\title{
Imagem Corporal e Hábitos Alimentares na Anorexia Nervosa: Uma Revisão Integrativa da Literatura
}

\author{
Body Image and Food Habits in Anorexia Nervosa: An Integrative Review \\ of the Literature
}

\author{
Carolina Leonidas* \& Manoel Antônio dos Santos \\ Universidade de São Paulo, Ribeirão Preto, Brasil
}

\begin{abstract}
Resumo
Revisão integrativa da literatura com objetivo de investigar os construtos da imagem corporal e hábitos alimentares na anorexia nervosa (AN). As bases consultadas foram MedLine, LILACS e PsycINFO, no período de 2005 a 2009. A maioria dos artigos encontrados é constituída de estudos não-experimentais e descritivos, provenientes de fontes internacionais. Os resultados evidenciaram que a personalidade de mulheres com AN é permeada por baixa autoestima, sentimentos de inferioridade, inadequação, insegurança, perfeccionismo e obsessividade, fatores que acarretam acentuada inibição e retraimento social, e que exercem influência na distorção da imagem corporal e na aquisição de hábitos alimentares disfuncionais. Como conclusão ressalta-se a necessidade de tratamento interdisciplinar, e de novos estudos experimentais e nacionais, que busquem compreender a relação entre os construtos.

Palavras-chave: Anorexia, transtornos alimentares, imagem corporal, hábitos alimentares, revisão da literatura.

Abstract

This is an integrative review of the literature that aims to investigate the constructs of body image and eating habits in anorexia nervosa (AN). The examined databases were MedLine, LILACS and PsycINFO in the period between 2005 and 2009. Most of the studies were non-experimental and descriptive, from international sources. The results showed that the personality of women with AN is permeated by low selfesteem, feelings of inferiority and inadequacy, insecurity, perfectionism and obsessiveness. These are factors that cause high inhibition and social withdrawal, and that influence body image distortion and the acquisition of dysfunctional eating habits. In conclusion, it is highlighted the need of an interdisciplinary treatment and the development of new national experimental studies seeking to understand the relationship between the constructs.

Keywords: Anorexia, eating disorders, body image, food habits, literature review.
\end{abstract}

De acordo com o DSM-IV-TR ${ }^{T M}$ - Manual Diagnóstico e Estatístico de Transtornos Mentais, $4^{\mathrm{a}}$ edição (Associação Americana de Psiquiatria, 2002), a anorexia nervosa (AN) é um transtorno alimentar (TA) caracterizado por recusa do indivíduo a manter o peso corporal na faixa normal mínima, de acordo com sua idade e altura. Também constituem critérios diagnósticos um temor intenso de ganhar peso e uma perturbação significativa na imagem corporal. Tais sintomas resultam em caquexia, isto é, fraqueza geral do corpo e má disposição corporal decorrente da desnutrição (Claudino \& Borges, 2002).

\footnotetext{
" O presente estudo recebeu apoio financeiro da Fundação de Amparo à Pesquisa no Estado de São Paulo (FAPESP), por meio da concessão de bolsa de Iniciação Científica à primeira autora, processo $\mathrm{n}^{\circ} 08 / 50869-5$. Endereço para correspondência: Avenida Sibipirunas, 312, Jardim Recreio, Ribeirão Preto, SP, Brasil 14040080. Tel.: (16) 3630-8350 e 9163-8929. E-mail: carol_leonidas@hotmail.com
}

Na concepção de Schilder (1935/1994), a imagem corporal é a representação mental que o indivíduo elabora de seu esquema corporal. Essa representação se organiza como um núcleo vital na constituição da personalidade, uma vez que corresponde a um conjunto funcional que permite que o indivíduo se diferencie do outro. Giordani (2006) sugere que a imagem que o indivíduo tem de seu próprio corpo é resultado de simbolizações e significações derivadas das interações cotidianas e das marcas afetivas, emocionais e socioculturais que são inscritas no corpo ao longo da vida.

O modo singular como cada sujeito configura sua imagem corporal, consciente ou inconscientemente, assume um significado crucial na manutenção da saúde e no desenvolvimento de sua corporeidade, motricidade e vitalidade, com reflexos na constituição da autoestima e nos relacionamentos afetivos e sociais. Por conseguinte, as perturbações graves na imagem corporal podem sinalizar a presença de psicopatologia (Peres \& Santos, 2007; 
Peres, Santos, \& Kruschewsky, 2007). Evidências científicas disponíveis na literatura indicam que a AN é um transtorno psicopatológico intimamente associado à grave distorção da imagem corporal (Bighetti et al., 2007; Oliveira \& Santos, 2006).

Entende-se como hábito, prática ou comportamento alimentar todas as formas de convívio com o alimento, que abarcam desde o momento da decisão de que alimento preparar, a forma de prepará-lo, os utensílios utilizados, as características, horários e divisão da alimentação em porções nas várias refeições do dia, certas preferências e aversões, até a digestão do alimento (Dunker \& Phillipi, 2003). Pode-se afirmar, portanto, que as práticas alimentares não se resumem apenas aos alimentos que são ingeridos ou que se deixa de ingerir, mas englobam também as regras, significados e valores que permeiam os diferentes aspectos relativos à prática de consumo alimentar (Alessi, 2006).

No caso de pacientes com $\mathrm{AN}$, a alteração dos hábitos alimentares inicia com um jejum progressivo, abolindo a princípio os alimentos calóricos, ao que se segue a restrição de outros grupos de alimentos, evoluindo para um rígido controle sobre o comportamento alimentar (Sicchieri, Santos, J. E. Santos, \& Ribeiro, 2007).

A forma como a distorção da imagem corporal se manifesta é de enorme interesse para a investigação científica dos TA. O presente estudo destaca como questão de pesquisa a suposição de que a distorção grave da imagem corporal está relacionada às alterações dos hábitos alimentares de pacientes com AN. Isso incluiria tanto os alimentos que são evitados, como o modo como esses pacientes se posicionam diante de algumas situações que envolvem a alimentação, o que requer minucioso exame dos sentimentos que permeiam a recusa alimentar.

Acredita-se que a compreensão da forma pela qual as pacientes com AN vivenciam sua imagem corporal pode propiciar a elaboração de estratégias mais efetivas de prevenção, diagnóstico e tratamento psicológico, colaborando no prognóstico não apenas no plano psíquico, como também nutricional. A partir do momento em que a pessoa acometida sente-se menos insatisfeita com seu corpo, suas atitudes alimentares podem, gradativamente, evoluir na direção de padrões alimentares mais saudáveis.

Ao examinar os estudos dedicados ao campo dos TA, constata-se que existe uma vasta literatura acerca da imagem corporal, assim como há inúmeros trabalhos que investigam o comportamento alimentar. Contudo, não se sabe se as investigações abordam esses construtos de modo isolado ou se contemplam as possíveis relações existentes entre a imagem do corpo e os hábitos alimentares.

Considerando esses argumentos, este estudo teve por objetivo investigar os construtos da imagem corporal e hábitos alimentares no contexto da $\mathrm{AN}$, identificando os aspectos que exercem influência direta na configuração desses construtos.

\section{Método}

Para alcançar o objetivo proposto empreendeu-se uma revisão integrativa da literatura científica nacional e internacional. Buscou-se evidenciar o número de trabalhos publicados na área e o perfil dos estudos, de modo a identificar as tendências apontadas por essas publicações, possibilitando maior direcionamento das pesquisas sobre o tema.

A revisão integrativa foi eleita como método de pesquisa porque permite sumarizar estudos já finalizados acerca da temática abordada. Além disso, a revisão integrativa, quando elaborada de forma crítica, mantém os mesmos padrões de rigor, clareza e replicabilidade das pesquisas primárias (Ganong, 1987). Segundo Fernandes (2000) a revisão integrativa possibilita, ainda, a construção de uma análise criteriosa da literatura científica, contribuindo para ampliar discussões sobre métodos e resultados de pesquisa, assim como prover reflexões e apontamentos valiosos para a realização de futuras pesquisas.

\section{Procedimento}

Embora os métodos para a condução de revisões integrativas variem, é preciso atentar para certos padrões a serem seguidos. No presente estudo considerar-se-ão os procedimentos preconizados por Ganong (1987), como o estabelecimento de critérios para inclusão e exclusão dos estudos publicados e análise crítica dos seus resultados. A partir dos procedimentos destacados por ScorsoliniComin e Santos (2008), na realização da presente revisão foram cumpridas as seguintes etapas: seleção da questão temática, estabelecimento dos critérios para a extração da amostra, análise e interpretação dos resultados, e apresentação da revisão.

Para a elaboração da revisão integrativa, foram selecionados os seguintes descritores, de acordo com o DeCS - Descritores em Ciências da Saúde: anorexia nervosa, transtornos da alimentação/eating disorders, imagem corporal/body image, hábitos alimentares/food habits. Tendo em vista que o propósito deste estudo era reunir artigos que apresentassem uma intersecção entre os temas propostos, as buscas nas bases de dados foram realizadas com os unitermos de maneira combinada.

Após o levantamento das publicações, os resumos foram lidos e analisados segundo os critérios de inclusão/ exclusão pré-estabelecidos, que serão descritos na sequência. Os trabalhos selecionados tiveram seus resumos relidos e analisados em profundidade. Em um segundo momento, os artigos foram recuperados na íntegra e analisados, constituindo o corpus de análise da revisão.

\section{Bases de Dados Consultadas}

Com vistas a assegurar uma abrangência da revisão, foram consultadas as seguintes bases de dados: MedLine, LILACS e PsycINFO. MedLine é uma base de dados internacional da área médica e biomédica, produzida pela National Library of Medicine (NLM) e que contém refe- 
rências bibliográficas e resumos de mais de 5.000 títulos de revistas científicas publicadas nos Estados Unidos e em outros 70 países. Contém referências de artigos publicados desde 1966 até o momento, que cobrem as áreas de: medicina, biomedicina, enfermagem, odontologia, veterinária e ciências afins. LILACS é uma base de dados cooperativa da Rede BVS, que compreende a literatura relativa às ciências da saúde, publicada nos países da América Latina e do Caribe a partir de 1982. Essa base alcança mais de 400.000 mil registros e contempla artigos de cerca de 1.300 revistas conceituadas da área da saúde, das quais aproximadamente 730 continuam sendo atualmente indexadas. Também abarca outros documentos, tais como teses, capítulos de teses, livros, capítulos de livros, anais de congressos ou conferências, relatórios técnico-científicos e publicações governamentais. Por fim, PsycINFO é uma base de dados da American Psychological Association (APA), que abrange artigos com temas relacionados à psicologia, educação, psiquiatria e ciências sociais. Disponibiliza publicações veiculadas em periódicos internacionais e contém aproximadamente 1.500 .000 registros.

Desse modo, ao considerar a especificidade do objeto de estudo, selecionou-se para o presente estudo uma base de dados internacional, que é considerada referência na literatura em saúde (MedLine), uma base bibliográfica que inclui os trabalhos publicados na América Latina e do Caribe (LILACS) e uma base, também de âmbito internacional, que engloba publicações específicas da Psicologia e áreas afins (PsycINFO).

\section{Critérios de Inclusão e Exclusão das Publicações}

Em relação ao período de publicação, circunscreveuse um intervalo de cinco anos. Assim, foram selecionados artigos publicados entre os anos de 2005 e 2009. Em relação ao idioma, restringiu-se a busca aos trabalhos publicados nos idiomas português, espanhol, inglês e francês. Os artigos repetidos, isto é, que apareceram mais de uma vez em diferentes combinações de palavras-chave, foram computados uma única vez.

Nesta revisão foram excluídos trabalhos como teses, dissertações, livros, capítulos de livros, resenhas, críticas, comentários, editoriais, anais e relatórios científicos. Essa escolha se deve ao fato de que, em uma das bases utilizadas (LILACS), notou-se o registro de trabalhos desse tipo. Como se sabe, essas produções não passam por rigoroso processo de avaliação por pares, nos moldes do peer review, procedimento que garante a qualidade do artigo e de sua apreciação científica. Desse modo, a fim de realçar apenas os trabalhos submetidos a processo rigoroso de avaliação, foram selecionados exclusivamente artigos científicos publicados em periódicos indexados nas bases bibliográficas selecionadas.

Foram excluídos ainda: estudos a respeito de condutas alimentares de populações específicas (por exemplo, atletas, bailarinas, modelos, universitários, drogaditos, entre outros), trabalhos que abordavam questões sobre conduta alimentar relacionada a outros transtornos (por exemplo, obesidade, câncer, aids, diabetes mellitus, hipertireoidismo, entre outros), temas muito distantes daqueles abrangidos pela presente revisão (por exemplo: dismorfia muscular, abuso de álcool), estudos de casos, validação de testes e outros instrumentos padronizados para países que não o Brasil, trabalhos que focalizam outros TA que não a $\mathrm{AN}$, investigações a respeito dos efeitos de determinados medicamentos. Excluíram-se também estudos voltados exclusivamente a questões biomédicas (neurobiologia da imagem corporal, por exemplo) e também investigações de outras áreas do saber, que não apresentavam relação direta com a Psicologia ou com o enfoque desta investigação.

Considerando esses pressupostos, os critérios de inclusão e exclusão previamente estabelecidos foram rigorosamente seguidos, tendo em vista a concisão e delimitação do campo.

\section{Resultados e Discussão}

A busca foi iniciada pela base de dados MedLine, na qual foi encontrado um total de 2104 artigos a partir dos diferentes agrupamentos das palavras-chave, sendo que 76 foram selecionados pela pertinência aos critérios de inclusão e exclusão. Destes estudos apenas seis foram recuperados na íntegra. A seguir foi realizada a busca na base de dados LILACS, na qual foi encontrado um total de 92 artigos a partir dos diferentes agrupamentos dos unitermos utilizados na pesquisa, sendo que 11 foram selecionados e seis recuperados. A base de dados consultada a seguir foi o PsycINFO, no qual foram encontrados 1037 artigos, sendo que 40 foram selecionados e 13 recuperados. Dos 127 artigos selecionados, após rigorosa aplicação dos critérios de inclusão, foram coligidos 25 trabalhos, que constituíram o corpus de análise que dá suporte à presente revisão. Esses dados podem ser melhor visualizados na Tabela 1 .

Tabela 1

Distribuição dos Artigos Segundo as Bases de Dados Bibliográficos Selecionadas

\begin{tabular}{lcccccc}
\hline Base & Total de artigos & $\%$ & Artigos selecionados & $\%$ & Artigos recuperados & $\%$ \\
\hline MedLine & 2104 & 65,1 & 76 & 59,8 & 6 & 24,0 \\
LILACS & 92 & 2,8 & 11 & 8,7 & 6 & 24,0 \\
PsycINFO & 1037 & 32,1 & 40 & 31,5 & 13 & 52,0 \\
\hline Total & 3233 & 100 & 127 & 100 & 25 & 100 \\
\hline
\end{tabular}


Leonidas, C. \& Santos, M. A. (2012). Imagem Corporal e Hábitos Alimentares na Anorexia Nervosa: Uma Revisão Integrativa da Literatura.

No que diz respeito às fontes de publicação, foi localizado um artigo em cada um dos seguintes periódicos científicos: Academic Psychiatry, Behavior Research and Therapy, Body Image, Comprehensive Psychiatry, Journal of Health Psychology, Psicologia \& Sociedade, Psychology and Behavior e Revista de Psiquiatria Clínica. Foram encontrados dois trabalhos no Journal of Social and Clinical Psychology, no International Journal of Mental Health Nursing e no International Journal of Eating Disorders, e três artigos nas revistas: Eating Behaviors, Medicina e European Eating Disorders Review. Observa-se um predomínio de periódicos internacionais, especialmente da América do Norte (40\%), sendo que destes, sete eram provenientes dos Estados Unidos e três do Canadá. Também houve um número expressivo de publicações de países europeus $(28 \%)$, sendo que destes a maioria era proveniente do Reino Unido (42,8\%), seguidos da Espanha, Itália e Suécia. Cinco (20\%) dos 25 estudos recuperados foram publicados em revistas científicas nacionais.

No que concerne ao período de publicação, o maior número de trabalhos publicados no tema investigado no presente estudo concentrou-se nos anos $2005(n=33) \mathrm{e}$ $2006(n=32)$, havendo diminuição gradual nos anos posteriores: $2007(n=27), 2008(n=21)$ e $2009(n=14)$. Esses dados sugerem que se trata de um tema que tem recebido atenção da comunidade científica na atualidade. Porém, a diminuição relativa do número de publicações nos anos recentes indica que o interesse da comunidade científica por esse problema de investigação tem arrefecido. Com base nesses dados, e levando-se em conta a importância do tema para melhor compreensão dos TA e, por conseguinte, para o aprimoramento dos serviços de saúde, pode-se inferir que novas investigações nesse campo do conhecimento ainda se fazem necessárias, especialmente no contexto nacional.

Circunscreveu-se o levantamento ao período de 2005 a 2009. Em virtude da quantidade de trabalhos encontrados e selecionados, optou-se por realizar a análise crítica apenas dos artigos recuperados na íntegra, excluindo-se desse exame os resumos. A observância rigorosa dos critérios de inclusão/exclusão fez com que o número de artigos recuperados (25) fosse consideravelmente menor do que os selecionados.

A maioria dos trabalhos recuperados corresponde a estudos empíricos não-experimentais, descritivos e exploratórios $(n=8)$ e revisões de literatura $(n=7)$, sendo uma com estudo de meta-análise (Cassin \& Von Ranson, 2005). Detectou-se também uma importante parcela de estudos experimentais $(n=7)$. Por fim, foi identificado apenas um estudo etnográfico e dois longitudinais (follow $u p$ ). De modo geral, as populações investigadas abrangeram: mulheres com TA, principalmente AN; adolescentes (principalmente do sexo feminino), mulheres jovens com AN, que já apresentaram o quadro ou que se encontravam em tratamento; mulheres com TA (grupo experimental) e sem TA (grupo controle); estudantes universitárias; pacientes em seguimento por programas de assistência multidisciplinar; mulheres com TA, com outros transtornos psiquiátricos e saudáveis; mulheres com AN, mulheres que faziam dieta e outras que não faziam; comparação entre estudantes universitários do sexo feminino e masculino. Seis estudos de revisão, dada sua natureza não empírica, não apresentaram participantes, e um não-experimental e descritivo também não trouxe descrição dos sujeitos. Nos 16 estudos empíricos restantes, observou-se uma enorme amplitude de variação das amostras: desde oito participantes (Giordani, 2006) a 1009 (Branco, Hilário, \& Cintra, 2006). As amostras clínicas variaram de oito (Giordani, 2006) a 333 pacientes com AN (Santonastaso, Zanetti, De Antoni, Tenconi, \& Favaro, 2006). A maior parte de estudos que incluíram grandes amostras investigou a população normal, em geral adolescentes e estudantes sem sintomas de TA.

No que diz respeito aos objetivos, os trabalhos revisados discorrem, de forma geral, sobre: quadro clínico e classificação dos TA; avaliação da imagem corporal (percepção, grau de satisfação, transtorno, déficit ou distorção, entre outras dimensões); manejo nutricional nos TA; cognições envolvidas nos comportamentos de bodychecking; relação entre TA e diferentes variáveis, como personalidade, autoestima, narcisismo, genética, normas culturais, mídia, identificação com pessoas do ambiente, outros sintomas psiquiátricos; avaliação das características clínicas e psicológicas de pacientes com AN que já tiveram bulimia nervosa (BN); exploração da experiência subjetiva de "sentir-se gorda"; investigação dos diversos fatores predisponentes aos TA em sua relação com as diferenças culturais.

Em relação aos instrumentos utilizados para o exame da imagem corporal e dos hábitos alimentares, na maioria dos estudos foram aplicadas escalas padronizadas para avaliação do componente de investigação. Os instrumentos mais utilizados foram: Eating Disorders Inventory (EDI), Eating Attitudes Test (EAT-26), Body Shape Questionnaire (BSQ), Body Checking Questionnaire (BCQ) e Body Checking Cognitions Scale (BCCS). Desses cinco questionários, quatro (EDI, EAT-26, BSQ e $\mathrm{BCQ}$ ) foram validados para a língua portuguesa. Apenas três estudos utilizaram a técnica de entrevista semiestruturada e um empregou a entrevista aberta, com foco na história de vida. Em sua maioria, utilizaram a avaliação do Índice de Massa Corporal (IMC) dos participantes de forma complementar à aplicação dos instrumentos padronizados.

Os resultados dos estudos revisados indicaram, de modo geral, que a personalidade das mulheres com AN é permeada por baixa autoestima, sentimentos de inferioridade e inadequação, insegurança, perfeccionismo e obsessividade, fatores que acarretam acentuada inibição e retraimento social (Borges, Sicchieri, Ribeiro, Marchini, \& Santos, 2006; Cassin \& Von Ranson, 2005; CastroFornieles et al., 2007; Nilsson, Sundboom, \& Häglöff, 2008; Peres \& Santos, 2006; Shea \& Pritchard, 2007). 
O funcionamento mental é marcado por compulsividade, emoções negativas, condutas evitativas e autopunição. Pacientes com AN se diferenciam de pacientes com BN no sentido de que as primeiras apresentam retraimento social, ausência de busca por sensações novas e evitação do contato interpessoal, ao passo que as segundas apresentam intensa impulsividade, busca exacerbada de estímulos e experiências novas, além de traços de personalidade borderline (Cassin \& Von Ranson, 2005). Ao considerar a complexidade que reveste essas características da estrutura e dinâmica de personalidade, os estudos demonstram, de modo consistente, a necessidade de tratamento interdisciplinar.

As adolescentes tendem a superestimar sua imagem corporal e se mostram mais insatisfeitas com a mesma, enquanto os meninos subestimam sua condição de sobrepeso (Branco et al., 2006). A imagem corporal é resultado da interação entre o indivíduo e seu meio social. A distorção dessa imagem inclui distúrbios da consciência cognitiva acerca do próprio corpo, alteração da consciência das sensações corporais, redução do senso de controle sobre as funções corporais e atribuição de razões afetivas para a realidade da configuração corporal. Esse distúrbio, ainda, a pensamento dicotômico, comparação equivocada, atenção seletiva e erro cognitivo. As imagens idealizadas de magreza expostas pela mídia levam a um aumento da insatisfação corporal e, consequentemente, a comportamentos alimentares disfuncionais (Monro \& Huon, 2006; Strahan, Spencer, \& Zana, 2007).

Ao considerar o construto da imagem corporal, a presente revisão evidenciou que existem algumas diferenças destacadas na literatura, principalmente no que diz respeito à abordagem teórica utilizada nos estudos que focalizam esse conceito. Tais diferenças conceituais precisam ser cuidadosamente consideradas, pois têm reflexos nos modelos de diagnóstico e intervenção terapêutica propostos. Giordani (2006) descreve a imagem corporal como resultado da intercomunicação entre o indivíduo e o mundo social no qual ele está inserido, considerando que a pessoa utiliza outras imagens, como a do corpo do outro, para definir a sua própria. Cooper, Deepak, Grocutt e Bailey (2007) sugerem que a experiência de "sentir-se gorda", vivenciada intensamente pelas pacientes com TA, parece estar relacionada a uma combinação de fatores, como angústia, sensações corporais internas e externas, sentimentos de rejeição, exclusão social, além de crenças negativas sobre si mesma.

Os trabalhos investigam a imagem corporal e sua associação com os TA a partir de vários pontos de vista: sociológico, comportamental e subjetivo - tanto intra como intersubjetivo. Vários autores associam o quadro de TA à imagem corporal negativa, permeada por sentimentos de inferioridade, inadequação e insegurança, bem como pelo perfeccionismo, obsessividade, compulsividade e emoções negativas (Borges et al., 2006; Cassin \& Von Ranson, 2005; Nilsson et al., 2008; Peres \& Santos, 2006).
A distorção da imagem corporal está frequentemente associada à característica de personalidade conhecida como perfeccionismo. O perfeccionismo, segundo Castro-Fornieles et al. (2007) e Nilsson et al. (2008), é importante componente na predisposição e na manutenção dos TA. O estudo dos primeiros autores referidos demonstrou que o perfeccionismo, além de estar muito relacionado à predisposição ao desenvolvimento dos TA, está presente em maior grau nesses quadros do que em outros transtornos psiquiátricos, como depressão e ansiedade. Nilsson et al. (2008) também perceberam que, quando o grau de perfeccionismo era alto, as pacientes demoravam mais para se recuperarem, sendo que o contrário também ocorria: menor perfeccionismo estava associado a melhor prognóstico. Assim, concluíram que, quanto maior o grau de perfeccionismo, pior é o prognóstico, o que é indício de que esse componente pode ser mais um fator preditor dos TA.

Outro componente importante nos TA e que tanto pode influenciar, como ser influenciado pela imagem corporal, é a autoestima (Shea \& Pritchard, 2007). Esses autores propõem que o déficit de autoestima depende da concomitância de outras variáveis para desencadear um TA, como o desejo de emagrecer e/ou a insatisfação corporal. Essas variáveis incluem: estresse, capacidade (ou incapacidade) de enfrentamento e perfeccionismo. Karpowicz, Skärsäter e Nevonen (2009), em seu estudo a respeito do efeito do tratamento sobre a melhora da autoestima (e outros aspectos relacionados ao TA) de pacientes com $\mathrm{AN}$, notaram que houve um aumento significativo nos indicadores após três meses de tratamento, o que sugere a relevância e eficácia do cuidado terapêutico.

Foi encontrado um único trabalho que buscou investigar os sentimentos despertados pelas experiências relacionadas ao corpo em mães com TA (Rørtveit, Aström, \& Severinsson, 2009). Esses autores realizaram entrevistas na modalidade de grupos focais, que abordavam problemas relacionados ao TA, culpa, vergonha e o significado de "ser mãe". A análise hermenêutica de conteúdo possibilitou a identificação de um tema principal: "sentimentos poderosos de sentir-se presa e com vergonha do próprio corpo", o que também foi colocado como parte do título do estudo. As experiências de vergonha e de "sentir-se presa", relacionadas ao modo como o corpo é vivenciado, foram relatadas como muito intensas. Uma implicação desse estudo para enfermeiras - já que se trata de um estudo realizado por enfermeiras psiquiátricas de um grupo de pesquisa em Saúde Mental da Mulher da Universidade de Stavanger, Suécia - é corroborar a importância de oferecer espaços dialógicos para que as mulheres possam articular suas dificuldades, especialmente aquelas ligadas aos sentimentos de "sentir-se presa" e com vergonha de seu próprio corpo. Os grupos de apoio com foco no tema dos TA podem ser considerados como forma de auxiliar essas mulheres e atenuar os sentimentos negativos relacionados ao quadro. 
Leonidas, C. \& Santos, M. A. (2012). Imagem Corporal e Hábitos Alimentares na Anorexia Nervosa: Uma Revisão Integrativa da Literatura.

Quatro dos estudos revisados trataram da influência da mídia e das normas culturais nos TA. Sabe-se que o ideal de magreza tem sido moldado pelo contexto social e se tornado cada vez mais difícil de atingir. Esse fato, associado ao fato de que a mídia é onipresente no imaginário contemporâneo e exerce influência sobre os processos de subjetivação, possibilita a compreensão do aumento da insatisfação corporal e dos consequentes comportamentos alimentares disfuncionais (Derenne \& Beresin, 2006). Confirmando esses dados, Strahan et al. (2007) demonstraram, em seu estudo, que mulheres expostas a imagens de mulheres magras comiam menos, e que esse dado mudava quando as mesmas eram expostas a imagens de mulheres com peso maior, porém mais bem-sucedidas. Ou seja, as normas culturais de beleza e sucesso parecem exercer influência sobre os comportamentos alimentares das mulheres. Monro e Huon (2006), em estudo que também colocava as participantes frente a imagens idealizadas retratadas pela mídia, também concluíram que a exposição a essas imagens pode levar a mudanças no comportamento alimentar. No entanto, Levine e Murnen (2009) sugerem que, apesar dos efeitos da mídia serem evidentes, é melhor considerá-la como um fator de risco que, mais tarde, pode ser tornar um fator causal dos TA.

Segundo Bergstrom e Neighbors (2006), as normas sociais estão relacionadas à ocorrência de uma variedade de comportamentos e atitudes, incluindo os relacionados à distorção da imagem corporal. Além disso, percepções equivocadas dessas normas, que podem ocorrer por vários motivos, também são responsáveis pela adoção de alguns comportamentos distorcidos. Os autores concluem que é necessário corrigir as percepções equivocadas das normas e, assim, diminuir os comportamentos problemáticos que podem dar início ao TA.

Entre os trabalhos que tratam da influência das normas culturais na apresentação dos TA, foi encontrado apenas um que investigou o impacto da aculturação, status socioeconômico, funcionamento familiar e controle psicológico em relação aos TA e à autoimagem em diferentes culturas. Soh, Touyz e Surgenor (2006) realizaram uma revisão da literatura a respeito desse tema e constataram que, dos estudos empíricos empreendidos, poucos investigaram hábitos alimentares patológicos de grupos de países distintos. Além disso, os resultados das pesquisas são variados e a questão do impacto das diferentes culturas nos TA continua obscura. A aculturação quase não foi levada em consideração nos estudos revisados e a relação entre TA em grupos de países não ocidentais e controle psicológico não foi verificada. Assim, o referido trabalho mostrou a necessidade de empreender mais estudos que abordem a influência que diferentes culturas podem exercer na predisposição aos TA.

Foram encontrados dois trabalhos, com dois autores em comum, que investigaram o construto de body checking. Esse conceito pode ser traduzido como "checagem corporal". Consiste no exame exagerado de partes do pró- prio corpo, motivado por uma preocupação intensa e persistente com a forma e o peso corporal. Seria, segundo Mountford, Raser e Waller (2008) e Waller, Sines, Meyer e Mountford (2008), uma forma de tentar controlar a distorção da imagem corporal. O primeiro trabalho evidenciou que há padrões específicos de associação entre os diversos elementos do narcisismo e diferentes aspectos do body checking, de forma que essa "checagem corporal" colabora com a função defensiva de manter a autoestima, ao invés de promover níveis positivos de autoestima narcísica. Esse dado parece ter relação com a distorção da imagem corporal encontrada em boa parte dos pacientes com TA, já que, ao realizarem a "checagem corporal", eles não se sentem felizes com o que vêem (Waller et al., 2008). O segundo estudo dedicado a esse tema, realizado por Mountford et al. (2008), demonstrou que pacientes com AN e que lançaram mão de métodos purgativos apresentaram poucos comportamentos de body checking e cognições relacionadas. No entanto, a crença de que o body checking possibilita ao indivíduo ter uma noção acurada do seu peso estava mais relacionada aos comportamentos purgativos do que ao diagnóstico propriamente dito. $\mathrm{O}$ estudo ressaltou a importância de novas pesquisas que busquem compreender a relação entre o fenômeno do body checking e mecanismos neurológicos.

Com relação aos hábitos alimentares, um dos trabalhos encontrados abordava o padrão de alimentação adotado por adolescentes. Branco et al. (2006) realizaram uma avaliação da percepção e satisfação que adolescentes tinham do seu próprio corpo, e investigaram a relação desses componentes com seu estado nutricional. A maior parte da população do estudo estava eutrófica, isto é, dentro dos limites de peso considerados normais para a idade, porém muitas meninas não se percebiam dessa forma: elas superestimavam sua imagem corporal. Já os meninos subestimavam sua condição de sobrepeso e obesidade. A insatisfação com a imagem corporal foi mais prevalente entre adolescentes com sobrepeso e obesidade, com destaque para as meninas, o que talvez as incentive a buscar formas de diminuir o peso, ou se manterem em eutrofia. Esse estudo corroborou achados anteriores de que meninas adolescentes podem constituir população de risco para ocorrência dos TA.

Ainda a respeito da relação entre satisfação corporal e comportamentos alimentares de adolescentes, Shroff e Thompson (2006) buscaram avaliar similaridades entre meninas que pertenciam a grupos de amizade e pessoas com as quais se identificavam, levando em conta as seguintes variáveis: insatisfação com a imagem corporal, desejo de emagrecer, comportamentos bulímicos e autoestima. Além disso, também examinaram os níveis de distorção dos padrões alimentares e da imagem corporal. Para alcançar esses objetivos foi aplicada uma bateria de testes e instrumentos de avaliação, cujos resultados indicaram que as participantes apresentavam similaridades significativas em relação às suas amigas no que diz res- 
peito à autoestima, mas não à imagem corporal ou TA. Outras similaridades encontradas foram: supressão de sentimentos, internalização do ideal de magreza e comparações entre si no que diz respeito à aparência. Ou seja, comprovou-se que o meio ambiente e as pessoas com quem as participantes costumavam conviver exerciam importante influência nos seus sentimentos nutridos em relação a si mesmas e aos seus corpos.

Sicchieri, Bighetti, Borges, Santos e Ribeiro (2006) conduziram um estudo a respeito do manejo nutricional nos TA, no qual ficou claro que o trabalho interdisciplinar desenvolvido por equipe de saúde é a melhor alternativa para se tratar pacientes e suas famílias. Essa clientela demanda cuidados especializados, mas ainda há muito para se descobrir para que se possa progredir na abordagem dos TA que envolvem complicações nutricionais muito complexas.

A respeito da ingestão calórica por parte de mulheres com AN do tipo purgativo, Burd et al. (2009) realizaram um estudo que teve como objetivo avaliar essa variável nos dias em que as participantes apresentavam comportamentos de compulsão e/ou purgação, e examinar o grau de intensidade desses comportamentos. Os resultados demonstraram que, nos dias em que os comportamentos compulsivos e purgativos ocorreram, as participantes relataram ingestão significativa de calorias quando comparada aos dias em que nenhum desses comportamentos ocorria, ou quando apenas um deles (episódios compulsivos ou purgativos) ocorria. Além disso, os autores também notaram que os episódios compulsivos apresentavam pouca intensidade nos dias em que a purgação não ocorria. A ingestão de energia, de forma geral, foi mais alta do que o esperado. Assim, os autores concluíram que a ingestão alimentar variava significativamente em função dos comportamentos alimentares compulsivos. Esses comportamentos eram por vezes tão intensos quanto aqueles relatados por participantes com $\mathrm{BN}$ em outros estudos.

Outro trabalho deu ênfase às pacientes com AN que apresentaram histórico de compulsão e purgação alimentar (Santonastaso et al., 2006). Dos 333 participantes, 7\% relataram histórico anterior de $\mathrm{BN}$ e foram comparados com um grupo controle de 48 pacientes sem esses antecedentes psiquiátricos. Desses $7 \%$, aqueles com diagnóstico de $\mathrm{BN}$ do tipo não purgativo apresentaram início tardio da doença, maior taxa de sintomas psiquiátricos, mais morbidade psiquiátrica na família e maiores índices de abuso sexual. Pacientes com BN do tipo purgativo não apresentaram nenhuma dessas variáveis. Assim, concluiuse que o histórico de compulsão e purgação alimentar em pacientes com TA, independentemente da idade em que o quadro se instala, está sempre associado com maiores taxas de psicopatologia do que as observadas em pacientes com AN do tipo restritivo que nunca tiveram antecedentes de períodos de compulsão e purgação.

Sloan, Mizes e Epstein (2005) examinaram empiricamente a classificação dos TA utilizando uma amostra de pacientes que estavam à procura de tratamento. Os resultados revelaram quatro grupos distintos, sendo que três deles assemelhavam-se às classificações diagnósticas de: $\mathrm{AN}$ do tipo restritivo, $\mathrm{BN}$ e transtorno de compulsão alimentar periódica (TCAP). O último grupo incluía pacientes cuja gravidade dos sintomas não se encontrava manifesta (sintomas subclínicos). Também foi possível perceber que não havia relação significativa entre os grupos empiricamente percebidos e os diagnósticos clínicos. Os autores consideram que é importante discutir as implicações desses achados no sistema de classificação diagnóstica e no tratamento dos TA.

Encontrou-se um estudo que partiu da hipótese de que não é o peso corporal que tem importância sobre os sintomas psicopatológicos de pacientes com TA, e sim seus comportamentos alimentares, uma vez que, segundo Zandian, Ioakimidis, Bergh e Södersten (2007), mais do que transtornos psiquiátricos, TA são TA propriamente ditos. Para testar essa hipótese, os autores treinavam as pacientes a comer e avaliavam sua alimentação por meio de uma escala conectada ao computador, denominada Mandometer, que delineava a curva de rendimento da taxa de alimentação. Foi possível perceber que os dois grupos investigados, constituídos por 27 pacientes com IMC < $14 \mathrm{~kg} / \mathrm{m}^{2}$ e 18 pacientes com IMC $>15,5 \mathrm{~kg} / \mathrm{m}^{2}$, apresentavam os mesmos padrões alimentares, com pouca quantidade de alimentos ingeridos e níveis elevados de sintomas psiquiátricos, incluindo o transtorno obsessivo compulsivo. Os autores perceberam que treinar os pacientes a ingerirem maiores quantidades de comida pode reverter esses sintomas. Após adotarem esse procedimento, os pacientes permanecem livres de sintomas durante um longo período de follow up. O estudo concluiu que o padrão de comportamento alimentar pode mediar a condição de fome-saciedade e a psicopatologia da AN.

Ao considerar que as percepções distorcidas da imagem corporal podem se constituir em fator desencadeador dos TA e que o padrão de comportamento alimentar é importante mediador desses quadros, a aplicação de estratégias de prevenção e intervenção focadas na redução da distorção da imagem corporal e na correção das alterações dos hábitos alimentares pode ser um esforço bastante válido.

Apenas dois dos 25 estudos recuperados fizeram uma articulação direta entre imagem corporal e hábitos alimentares. Os achados dessas investigações indicaram que há relação positiva entre os dois construtos, ou seja, quanto maior a distorção da imagem corporal, maior a disfunção alimentar. Desse modo, a presente revisão evidenciou a necessidade de novas investigações acerca da temática, que permitam compreender a relação entre os construtos, de modo a subsidiar propostas de intervenção e prevenção em saúde.

\section{Considerações Finais}

O estudo de revisão demonstrou que existe uma literatura consolidada acerca da interface da imagem corporal 
Leonidas, C. \& Santos, M. A. (2012). Imagem Corporal e Hábitos Alimentares na Anorexia Nervosa: Uma Revisão Integrativa da Literatura.

e hábitos alimentares. No entanto, poucos estudos relacionam diretamente os dois construtos. Além disso, parte substancial dos trabalhos abordava os temas investigados nesta revisão relacionando-os com outros quadros que não a $\mathrm{AN}$ (por exemplo, obesidade, outros transtornos psiquiátricos) e outra parte utilizava amostras bem diversificadas (atletas, bailarinas, modelos, universitários, drogaditos, entre outras), distantes daquela amostra proposta na presente revisão.

Houve predomínio de trabalhos publicados em periódicos internacionais, principalmente nos Estados Unidos e em países europeus, com destaque para o Reino Unido. Esse dado evidencia a necessidade de incrementar investimentos em publicações brasileiras acerca da avaliação psicológica da imagem corporal e dos hábitos alimentares em pacientes com TA. Também são necessários estudos com níveis mais sofisticados de evidência, tais como os experimentais e quase-experimentais.

Nesse panorama destacamos a necessidade de futuras investigações acerca da temática, com o objetivo de fornecer esclarecimentos mais pormenorizados a respeito da imagem corporal na interface com os hábitos alimentares no contexto dos TA, já que as evidências sugerem que a melhora da distorção dessa imagem pode levar à redução dos hábitos alimentares disfuncionais e dos rituais purgativos. Acredita-se que a ampliação do número de publicações qualificadas nessa área poderá proporcionar maiores evidências para a prática clínica, levando a uma compreensão mais abrangente por parte dos profissionais envolvidos na assistência, sem esquecer da prevenção e promoção de saúde.

\section{Referências}

Alessi, N. P. (2006). Conduta alimentar e sociedade. Revista Medicina, 39(3), 327-332.

Associação Americana de Psiquiatria. (2002). DSM-IV-TR ${ }^{T M}$ Manual diagnóstico e estatístico de transtornos alimentares: Texto revisado (4. ed. rev., C. Dornelles, Trad.). Porto Alegre, RS: Artmed.

Bergstrom, R. L., \& Neighbors, C. (2006). Body image disturbance and the social norms approach: An integrative review of the literature. Journal of Social and Clinical Psychology, 25(9), 975-1000.

Borges, N. J. B. G., Sicchieri, J. M. F., Ribeiro, R. P. P., Marchini, J. S., \& Santos, J. E. (2006). Transtornos alimentares: Quadro clínico. Revista Medicina, 39(3), 340-348.

Bighetti, F., Santos, M. A., Ribeiro, R. P. P., Oliveira, E. A., Unamuno, M. R. L., \& Dos Santos, J. E. (2007). Transtornos alimentares: Anorexia e bulimia nervosas: Uma abordagem didática. In Associação Brasileira de Enfermagem, Programa de Atualização em Enfermagem: Saúde do adulto: Ciclo 2, Módulo 2 (pp. 9-44). Porto Alegre, RS: Artmed.

Branco, L. M., Hilário, M. O. E., \& Cintra, I. P. (2006). Percepção e satisfação corporal em adolescentes e a relação com seu estado nutricional. Revista de Psiquiatria Clínica, 33(6), 291-296.
Burd, C., Mitchell, J. E., Crosby, R. D., Engel, S. G., Wonderlich, S. A., Le Grange, D., et al. (2009). An assessment of daily food intake in participants with anorexia nervosa in the natural environment. International Journal of Eating Disorders, 42(4), 371-374.

Cassin, S. E., \& Von Ranson, K. M. (2005). Personality and eating disorders: A decade in review. Clinical Psychology Review, 25(7), 895-916.

Castro-Fornieles, J., Gual, P., Lahortiga, F., Gila, A., Casulà, V., Fuhrmann, C., et al. (2007). Self-oriented perfectionism in eating disorders. International Journal of Eating Disorders, 40(6), 562-568.

Claudino, A. M., \& Borges, M. B. F. (2002). Critérios diagnósticos para os transtornos alimentares: Conceitos em evolução. Revista Brasileira de Psiquiatria, 24(3), 7-12.

Cooper, M. J., Deepak, K., Grocutt, E., \& Bailey, E. (2007). The experience of 'feeling fat' in women with anorexia nervosa, dieting and non-dieting women: An exploratory study. European Eating Disorders Review, 15(5), 366-372.

Derenne, J. L., \& Beresin, E. V. (2006). Body image, media, and eating disorders. Academic Psychiatry, 30(3), 257-261.

Dunker, K. L., \& Phillipi, S. T. (2003). Hábitos e comportamentos alimentares de adolescentes com sintomas de anorexia nervosa. Revista de Nutrição, 16(1), 51-60.

Fernandes, L. M. (2000). Úlcera de pressão em pacientes críticos hospitalizados: Uma revisão integrativa da literatura. (Dissertação de Mestrado não-publicada). Universidade de São Paulo, Ribeirão Preto, SP.

Ganong, L. H. (1987). Integrative reviews of nursing research. Research in Nursing \& Health, 10(1), 1-11.

Giordani, R. C. F. (2006). A auto-imagem corporal na anorexia nervosa: Uma abordagem sociológica. Psicologia e Sociedade, 18(2), 81-88.

Karpowicz, E., Skärsäter, I., \& Nevonen, L. (2009). Self-esteem in patients treated for anorexia nervosa. International Journal of Mental Health Nursing, 18(5), 318-325.

Levine, M. P., \& Murnen, S. K. (2009). "Everybody knows that mass media are/are not [pick one] a cause of eating disorders": A critical review of evidence for a causal link between media, negative body image, and disordered eating in females. Journal of Social and Clinical Psychology, 28(1), 9-42.

Monro, F. J., \& Huon, G. F. (2006). Media-portrayed idealized images, self-objetification, and eating behavior. Eating Behaviors, 7(4), 375-383.

Mountford, V., Haase, A. M., \& Waller, G. (2008). Is body checking in the eating disorders more closely related to diagnosis or to symptom presentation? Behaviour Research and Therapy, 45(11), 2704-2711.

Nilsson, K., Sundbom, E., \& Häglöf, B. (2008). A longitudinal study of perfectionism in adolescent onset anorexia nervosa - Restricting type. Europen Eating Disorders Review, 16(5), 386-394.

Oliveira, E. A., \& Santos, M. A. (2006). Perfil psicológico de pacientes com anorexia e bulimia nervosas: A ótica do psicodiagnóstico. Revista Medicina, 39(3), 353-360.

Peres, R. S., \& Santos, M. A. (2006). Contribuições do Desenho da Figura Humana para a avaliação da imagem corporal na anorexia nervosa. Revista Medicina, 39(3), 361-370.

Peres, R. S., \& Santos, M. A. (2007). Imagem corporal: Conceitualização e avaliação. In P. W. Schelini (Ed.), Domínios da avaliação psicológica (pp. 125-139). Campinas, SP: Alínea. 
Peres, R. S., Santos, M. A., \& Kruschewsky, M. S. L. B. (2007). Imagem corporal em obesos mórbidos antes e depois de uma intervenção multidisciplinar. Psychologica, 44, 467-488.

Rørtveit, K., Aström, S., \& Severinsson, E. (2009). The feeling of being trapped in and ashamed of one's own body: A qualitative study of women who suffer from eating difficulties. International Journal of Mental Health Nursing, 18(2), 91-99.

Santonastaso, P., Zanetti, T., De Antoni, C., Tenconi, E., \& Favaro, A. (2006). Anorexia nervosa patients with a prior history of bulimia nervosa. Comprehensive Psychiatry, 47(3), 519-522.

Schilder, P. (1994). A imagem do corpo: As energias construtivas da psique (R. Wertman, Trad). São Paulo, SP: Martins Fontes. (Original work published 1935)

Scorsolini-Comin, F., \& Santos, M. A. (2008). Felicidade e bemestar subjetivo na literatura científica: Panorama histórico e perspectivas no período de 1970 a 2007 [CD ROM]. In $X$ Encontro de Pesquisadores em Saúde Mental da Escola de Enfermagem de Ribeirão Preto, Universidade de São Paulo, Ribeirão Preto, SP.

Shea, M. E., \& Pritchard, M. E. (2007). Is self-esteem the primary predictor of disordered eating? Personality and Individual Differences, 42(8), 1527-1537.

Sicchieri, J. M. F., Santos, M. A., Santos, J. E. \& Ribeiro, R. P. P. (2007). Avaliação nutricional de portadores de transtorno alimentar: Resultados após a alta hospitalar. Ciência, Cuidado e Saúde, 6(1), 68-75.

Sicchieri, J. M. F., Bighetti, F., Borges, N. J. B. G., Santos, J. E., \& Ribeiro, R. P. P. (2006). Manejo nutricional nos transtornos alimentares. Revista Medicina, 39(3), 371-374.

Sloan, D. M., Mizes, J. S., \& Epstein, E. M. (2005). Empirical classification of eating disorders. Eating Behaviors, 6(1), 53-62.

Soh, N. L., Touyz, S. W., \& Surgenor, L. J. (2006). Eating and body image disturbances across cultures: A review. European Eating Disorders Review, 14(1), 54-65.

Shroff, H., \& Thompson, J. K. (2006). Peer influences, bodyimage dissatisfaction, eating dysfunction and self-esteem in adolescent girls. Journal of Health Psychology, 11(4), 533551.

Strahan, E. J., Spencer, S. J., \& Zanna, M. P. (2007). Don't take another bite: How sociocultural norms for appearance affect women's eating behavior. Body Image, 4(4), 331-342.

Waller, G., Sines, J., Meyer, C., \& Mountford, V. (2008). Body checking in the eating disorders: Association with narcissistic characteristics. Eating Behaviors, 9(2), 163-169.

Zandian, M., Ioakimidis, I., Bergh, C., \& Söderten, P. (2007). Cause and treatment of anorexia nervosa. Physiology \& Behaviour, 92(1-2), 283-290. 